\title{
Efficient Data Reduction at the Edge of Industrial Internet of Things for PMSM Bearing Fault Diagnosis
}

\author{
Xiaoxian Wang $\left.{ }^{(}\right)$, Siliang Lu ${ }^{\circledR}$, Senior Member, IEEE, Wenbin Huang ${ }^{\circledR}$, Member, IEEE, \\ Qunjing Wang ${ }^{\circledR}$, Member, IEEE, Shiwu Zhang ${ }^{\circledR}$, Member, IEEE, and Min Xia ${ }^{\circledR}$, Member, IEEE
}

\begin{abstract}
An efficient data reduction algorithm is designed and implemented on an industrial Internet of Things (IIoT) node for permanent magnet synchronous motor (PMSM) bearing fault diagnosis in variable speed conditions. Leakage flux and vibration signals are, respectively, acquired by a magnetic sensor and an accelerometer on the IIoT node in a noninvasive manner. These two signals are processed and mixed on the IIoT and transmitted to a server. The received signal is separated, the cumulative rotation angle is calculated, and the vibration signal is resampled for bearing fault identification. The proposed method can reduce about $95 \%$ of the transmission data while maintaining sufficient precision in bearing fault diagnosis in comparison to a traditional method. The proposed method based on edge computing reduces the power consumption, and hence it is suitable to use on a battery-supplied IIoT node for remote PMSM condition monitoring and fault diagnosis.
\end{abstract}

Index Terms-Bearing fault diagnosis, data reduction, edge computing, IIoT, multiple sensor signal fusion, permanent magnet synchronous motor (PMSM).

Manuscript received October 27, 2020; revised December 28, 2020; accepted January 3, 2021. Date of publication January 14, 2021; date of current version February 2, 2021. This work was supported in part by the Open Project Program of the State Key Laboratory of Mechanical Transmissions of Chongqing University under Grant SKLMT-KFKT-201812, in part by the National Natural Science Foundation of China under Grant 52075002, in part by the Open Project Program of the Traction Power State Key Laboratory of Southwest Jiaotong University under Grant TPL1905, and in part by the Natural Science Basic Research Program of Shaanxi under Grant 2019JM341. The Associate Editor coordinating the review process was Qiang Miao. (Corresponding authors: Siliang Lu; Shiwu Zhang.)

Xiaoxian Wang is with the State Key Laboratory of Mechanical Transmission, Chongqing University, Chongqing 400044, China, also with the College of Electronics and Information Engineering, Anhui University, Hefei 230601, China, and also with the Department of Precision Machinery and Precision Instrumentation, University of Science and Technology of China, Hefei 230027, China (e-mail: wxx612@mail.ustc.edu.cn).

Siliang Lu is with the State Key Laboratory of Mechanical Transmission, Chongqing University, Chongqing 400044, China, also with the College of Electrical Engineering and Automation, Anhui University, Hefei 230601, China, and also with the Traction Power State Key Laboratory, Southwest Jiaotong University, Chengdu 610031, China (e-mail: lusliang@mail.ustc.edu.cn).

Wenbin Huang is with the State Key Laboratory of Mechanical Transmission, Chongqing University, Chongqing 400044, China (e-mail: whuang@cqu.edu.cn).

Qunjing Wang is with the National Engineering Laboratory of Energy-Saving Motor and Control Technology, College of Electrical Engineering and Automation, Anhui University, Hefei 230601, China (e-mail: wangqunjing@ahu.edu.cn).

Shiwu Zhang is with the CAS Key Laboratory of Mechanical Behavior and Design of Materials, Department of Precision Machinery and Precision Instrumentation, University of Science and Technology of China, Hefei 230027, China (e-mail: swzhang@ustc.edu.cn).

Min Xia is with the Department of Engineering, Lancaster University, Lancaster LA1 4YW, U.K. (e-mail: m.xia3@lancaster.ac.uk).

\section{INTRODUCTION}

$\mathbf{S}$ ERVO motors are one of the most pivotal actuators in a smart factory in the current Industry 4.0 era [1]. Permanent magnet synchronous motors (PMSMs) are extensively used in the servo system because of their distinct merits, including high efficiency, power density, low noise, vibration, and torque ripple [2]. However, motor performance degenerates after a long time of operation. The degenerated motor produces extra heat and vibration, which affect the motion accuracy and even cause damages or accidents [3]. Thus, condition monitoring and fault diagnosis of PMSM motors can guarantee the safe operation of the production lines, reduce maintenance costs, and avoid potential hazards [4].

Industrial Internet of Things (IIoT) is an emerging technique that has been widely used in machine condition monitoring and fault diagnosis. The IIoT nodes are installed at proper positions of the machine systems, and the acquired signals from the decentralized nodes are transmitted to the router and then to the data center or cloud for further processing. The IIoT technique provides a convenient and flexible mode for networking as it does not require complicated cable wiring. The IIoT nodes can also be easily added, removed, and replaced following the requirements of condition monitoring [5].

With an increasing number of IIoT nodes being deployed, data explosion becomes a new challenge [6]. The rapid-growth data occupy the transmission channels and stress the servers with limited storage and computing resources. Edge computing is an emerging computing paradigm that could release the stress of centralized computing by allocating the computing on the edges of the networks. Edge computing has distinct merits including high efficiency, low latency, and huge connections [7]. If the motor signals can be pre-processed using the edge computing technique, the agility and efficiency of condition monitoring and fault diagnosis will be improved.

A bearing fault is one of the typical mechanical faults in a motor [8]-[10]. A statistical survey demonstrated that bearing faults accounted for almost $40 \%-50 \%$ of motor faults [11]. Thus, condition monitoring and fault diagnosis of PMSM bearing is an important part of a smart factory [12]. Vibration signals from an accelerometer can reflect the healthy condition of motor bearing. Accordingly, vibration signal analysis methods have been extensively investigated and applied. Fig. 1 shows 


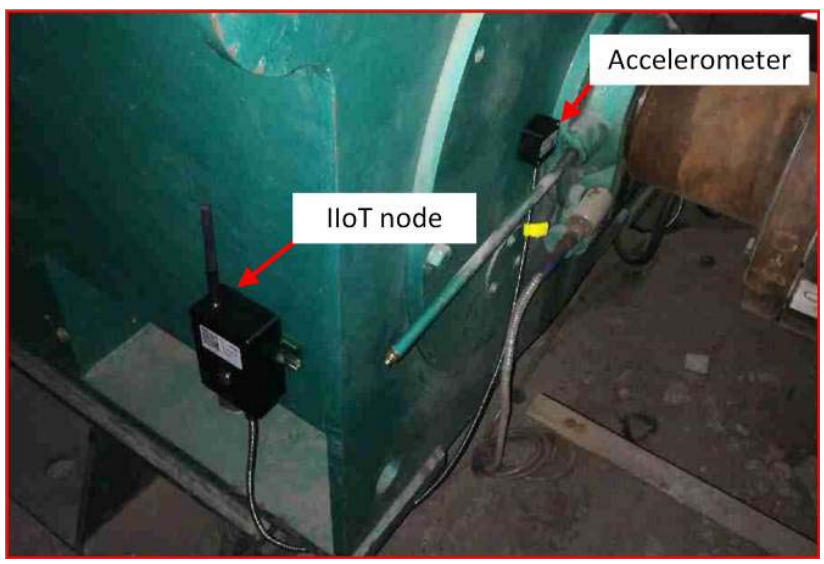

Fig. 1. Motor condition monitoring using an IIoT node.

an IIoT node that acquires the vibration signal for motor bearing condition monitoring.

One of the most crucial steps for fault diagnosis is the extraction of sensitive features related to bearing faults [13]-[18]. For instance, informative sets of features from a raw vibration signal are extracted for motor bearing fault diagnosis [19]. A method combining feature denoising and nearest-farthest distance preserving projection is proposed for machine fault diagnosis [20]. Statistical classification-based signal decomposition algorithm and time-frequency analysis are proposed to extract the vibration features for motor condition monitoring [21]. The majority of the feature extraction methods focus on the stationary signal analysis, which means that the motor rotation speed is constant or quasi-constant [22].

In a servo system, a PMSM usually works at variable speed conditions. The rotation speed is a nonlinear function to time [23]. The bearing fault signatures are highly related to the rotation speed. Hence, the speed information is necessary for bearing fault diagnosis under variable speed conditions [24]-[26]. Fig. 2 shows a typical PMSM servo system monitored by two IIoT nodes. The motor and driver are connected by the power and signal cables, and the distance between them is approximately 3-10 $\mathrm{m}$. The rotation speed signal can only be accessed from the connector of the motor driver because no interface is exposed on the motor side. Hence, two IIoT nodes are necessary to acquire the vibration and rotation speed signals synchronously, as indicated in Fig. 2. This configuration increases the facility cost and system complexity.

Generally, the IIoT nodes designed for motor condition monitoring acquire the raw data and transmit them to the server for further analysis. To guarantee the accuracy of condition monitoring, the signal should be of sufficient length. When an IIoT is powered by a battery, transmitting a long-length signal requires considerable energy and shortens the battery life. Given this, an efficient data reduction method is proposed and implemented on the IIoT node to improve the efficiency of data transmission in this study. The designed IIoT node acquires the magnetic and vibration signals synchronously without access to the motor control system. The signals are preprocessed, mixed, and then transmitted to the

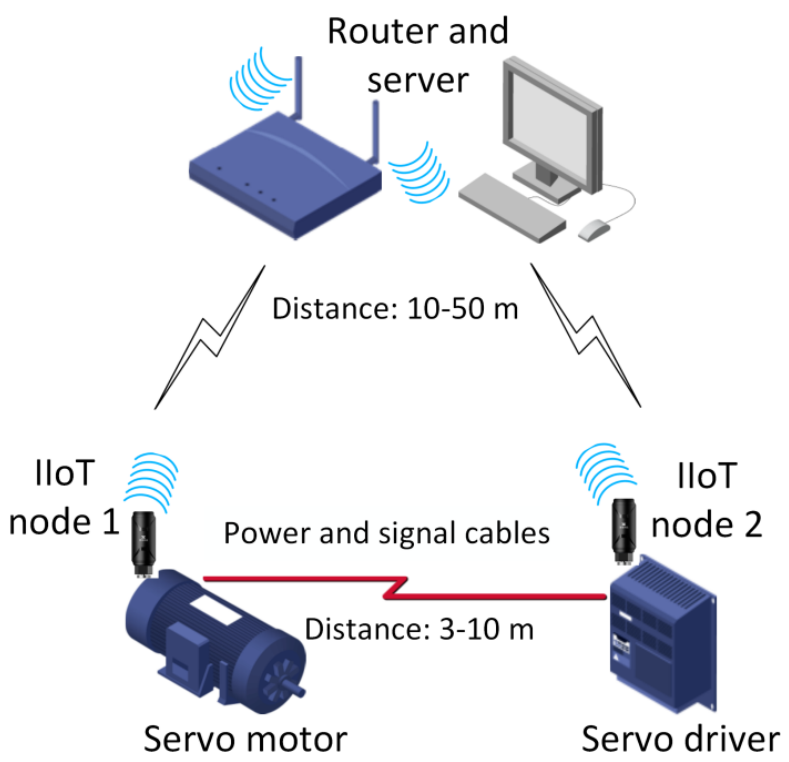

Fig. 2. Illustration of vibration and rotation speed measurement using two IIoT nodes.

server. The received signal is separated, and then the rotation speed is estimated from the magnetic signal. The vibration signal is resampled using the rotation speed, and the PMSM bearing fault can be diagnosed.

The contributions of this study are summarized as follows: 1) an IIoT node that can synchronously acquire the vibration and rotation speed signals is designed; 2) the signals are obtained in a noninvasive manner, which avoids complicated cable wiring and sensor installation; 3 ) the proposed method can significantly reduce the data to be transmitted, and then reduce the occupation of transmission channel, data storage space, and energy consumption; and 4) the factors that affect the performances along with the prospects of the method, algorithm, and hardware are discussed.

The rest of the study is organized as follows. Section II introduces the measurement principle and the hardware design of the IIoT node. Section III introduces the proposed data reduction method. Section IV presents the experimental setup. Section V presents the experimental results. Section VI evaluates the performances of the algorithm and hardware. Section VII discusses the future works, and Section VIII draws the conclusions of this study.

\section{Principle and Hardware Design OF THE IIOT NODE}

A data reduction method is proposed and implemented on a designed IIoT node to improve the data transmission efficiency and motor fault diagnosis accuracy. The method principle and hardware design are introduced as follows.

\section{A. Principle of the Proposed Method}

Fig. 3 shows the measurement principle of the proposed method. A PMSM consists of a spinning rotor, a stator, and two bearings installed at the two ends of the rotor shaft. The vibration signal is acquired by a micro-electromechanical 


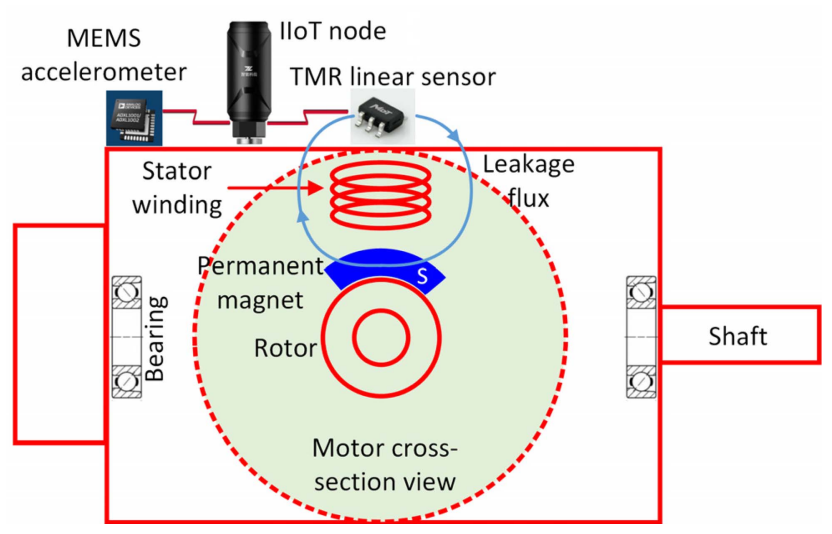

Fig. 3. Principle of the proposed method using only one IIoT node.

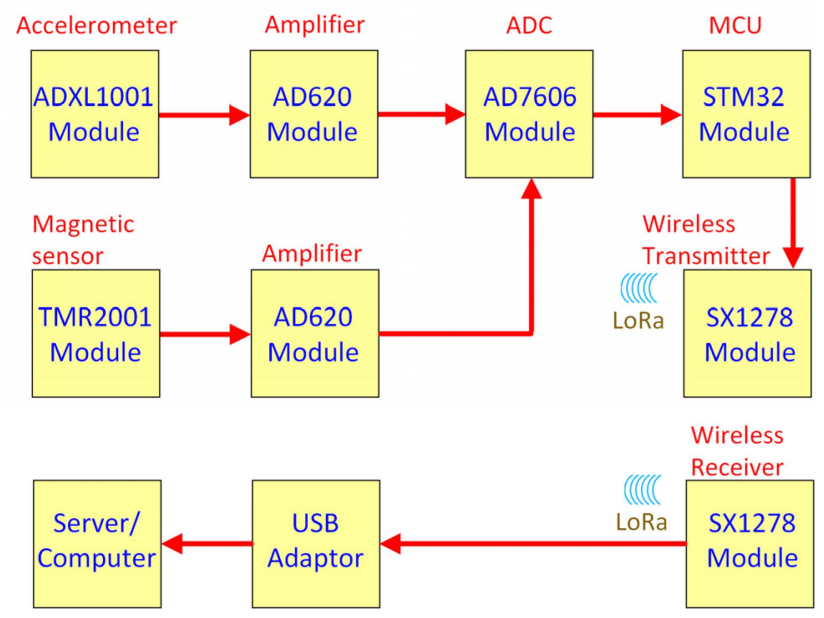

Fig. 4. Connections of components in the IIoT node and server.

systems (MEMS) accelerometer installed on the motor housing and the radial direction of the test bearing. When the test bearing has a localized fault on the outer raceway, inner raceway, or rollers, the collisions are generated when a roller rolls over the defect points. The fault-induced impulses propagate to the motor housing and are captured by the accelerometer.

The magnetic signal used for rotation speed measurement is acquired by a tunnel magnetoresistance (TMR) linear sensor installed on the center area of the motor housing. The cross-sectional view of the PMSM is shown within the motor profile in Fig. 3. The motor rotor mounted by the permanent magnets is pulled by the rotational flux generated from the stator winding. The dominant air gap between the rotor and stator leaks toward the outside of the motor housing, and then the leakage flux is captured by the sensitive TMR sensor. The vibration and magnetic signals are acquired and processed by an IIoT node and then transmitted to a server for bearing fault diagnosis.

\section{B. Hardware Design of the IIoT Node}

The hardware circuit of an IIoT node prototype is designed to realize efficient data reduction and bearing fault diagnosis, as shown in Fig. 4. The vibration signal is acquired by an MEMS accelerometer (ADXL1001, Analog Device Inc.).

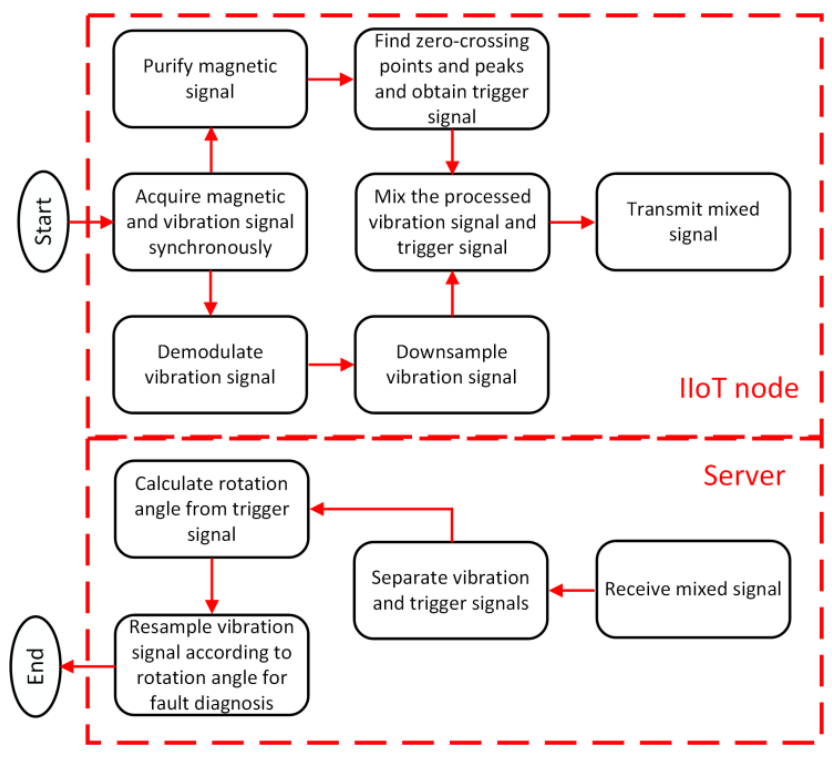

Fig. 5. Algorithm flowchart of the IIoT node.

The bandwidth, full range, and sensitivity of this accelerometer are $0-11 \mathrm{kHz}, \pm 100 \mathrm{~g}$, and $20 \mathrm{mV} / \mathrm{g}$, respectively. The leakage flux is acquired by a magnetic sensor (TMR2001, MultiDimension Technology, Inc.). The linear range and sensitivity of this sensor are \pm 25 Gauss and $8 \mathrm{mV} /$ Gauss, respectively. The weak analog output voltages of the sensors are amplified by an instrument amplifier (AD620, Analog Device Inc.).

The amplified signals are sampled and quantized by an analog-to-digital converter (ADC; AD7606, Analog Device Inc.). The resolution and the maximal synchronous sampling frequency of the ADC are 16-bit and $200 \mathrm{kHz}$, respectively. The converted digital signals are processed by a microcontroller unit (MCU; STM32F407, STMicroelectronics Inc.). The clock frequency and the flash memory of the MCU are $168 \mathrm{MHz}$ and $1 \mathrm{MB}$, respectively. The processed signal is transmitted to a server through a wireless transmitter (SX1278, SEMTECH Inc.) that works at $433 \mathrm{MHz}$, and the maximal programmable bit rate is up to $300 \mathrm{kbps}$ [27]. To guarantee stable transmission, the Baud rate is set as 19,200 bps in this study. The transmitted signal is received by a paired wireless receiver which connects to a USB adaptor. The signal is finally recorded by a data acquisition software on a server/computer, as shown in Fig. 4.

\section{EfFicient Data Reduction Method for PMSM BEARING FAUlt Diagnosis}

In this section, the data reduction algorithms for PMSM bearing fault diagnosis are introduced, as shown in Fig. 5. The algorithms consist of the IIoT node module and server module.

\section{A. Magnetic Signal Processing}

The discrete forms of the vibration and magnetic signals are, respectively, denoted as $V[n]$ and $M[n], n=1,2$, $\ldots, N$, where $N$ is the signal length. The leakage flux signal is generally weak and corrupted by background noise, and 
hence the magnetic signal is purified to estimate the rotation angle of the motor shaft accurately [28]. A bandpass infinite impulse response filter is used to purify the magnetic signal as follows [29]:

$$
F M[n]=-\sum_{k=1}^{K_{1}} a_{k} F M[n-k]+\sum_{k=0}^{K_{2}} b_{k} M[n-k]
$$

where $F M[n]$ is the filtered signal, $a_{k}$ and $b_{k}$ are the filter parameters, and $K_{1}$ and $K_{2}$ are integers. These parameters are determined by the type, order, and bandwidth of the filter. After signal filtering, the magnetic signal becomes a quasi-sinusoid with varying frequencies due to motor speed variation.

The key phases of the filtered magnetic signal are extracted using the zero-crossing detection and peak detection methods to reduce the signal length to be transmitted, as shown in Algorithm 1. By executing Algorithm 1, four key phases at $\pi / 2, \pi, 3 \pi / 2$, and $2 \pi$ are positioned. The resolution of the mechanical angle can be denoted as follows [30]:

$$
A R=\frac{360^{\circ}}{4 \times p}
$$

where $p$ is the number of pole pairs of the PMSM.

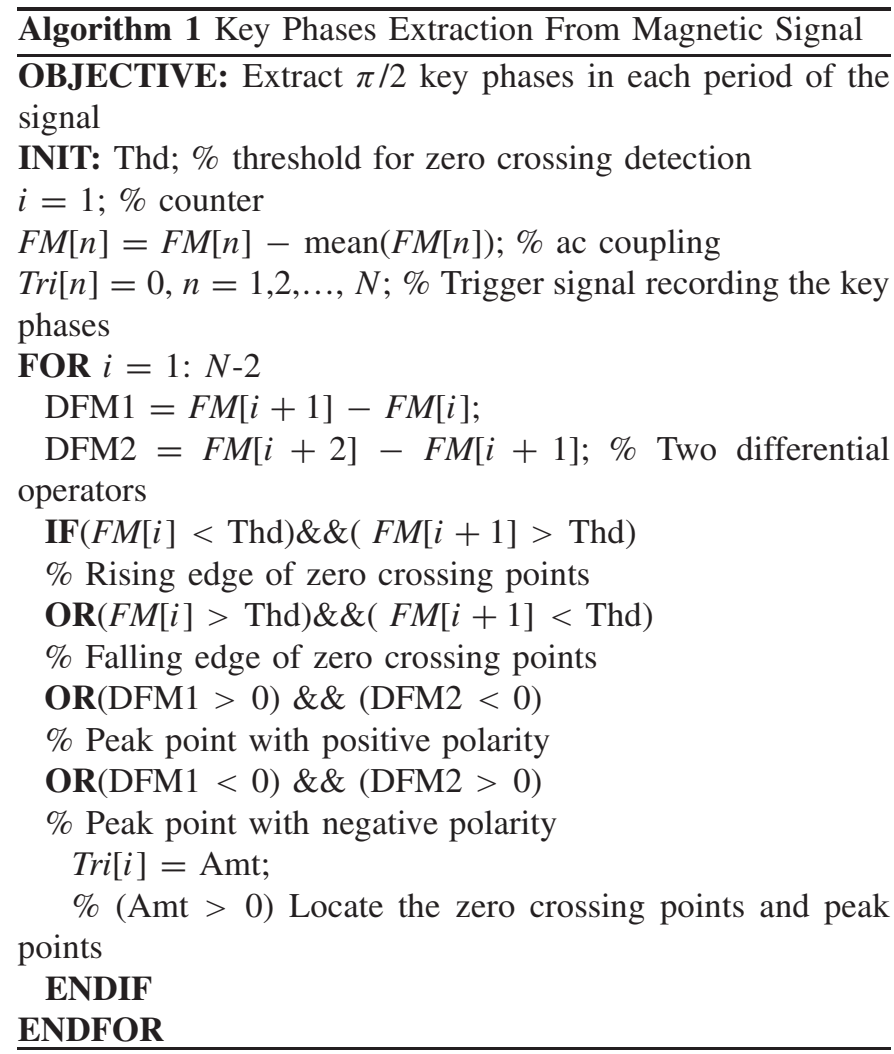

\section{B. Vibration Signal Processing}

When a fault occurs in a bearing, the PMSM is excited by the fault-induced collisions. The vibration signal is a modulated signal in which the resulting frequency is modulated by the machine resonance. The resonance frequency can be thousands of Hertz, and the sampling frequency should be multiple times the resonance frequency according to the Nyquist sampling theorem. In practice, resonance demodulation is commonly used for bearing fault detection [31]. By demodulating the vibration signal, the fault signatures are revealed in the low-frequency region (a few hundred Hertz) in the envelope spectrum.

Accordingly, the vibration signal is demodulated on the IIoT. Then, the demodulated signal can be downsampled and transmitted. This signal pre-processing step can reduce the length of the signal to be transmitted. Here, the vibration signal is processed using an amplitude demodulation method. The continuous form of the vibration signal $V(t)$ is used to illustrate the demodulation procedure.

The fault characteristic frequency and the machine resonance frequency are assumed as $f_{c}$ and $f_{r}$, respectively. Generally, these two frequencies satisfy $f_{r} \gg f_{c}$. Thus, the ideal vibration signal is expressed as follows [29]:

$$
\begin{aligned}
V(t) & =V_{c}(t) \times V_{r}(t) \\
& =\left[A_{c} \cos \left(2 \pi f_{c} t\right)+\text { Bias }\right] \times A_{r} \cos \left(2 \pi f_{r} t\right)
\end{aligned}
$$

where $V_{c}(t)$ and $V_{r}(t)$ are the two signal components induced by bearing fault and machine resonance, respectively, $a c$ and $A_{r}$ denote the corresponding amplitudes, and Bias is a constant number that guarantees $V_{c}(t)$ is a unipolar signal with a positive amplitude. Next, signal $V(t)$ is multiplied by itself and a constant value of 2 as follows:

$$
\begin{aligned}
& V^{\prime}(t) \\
&= 2 V^{2}(t) \\
&= \frac{1}{2} A_{c}^{2} A_{r}^{2}\left[1+\cos \left(4 \pi f_{c} t\right)\right]+\frac{1}{2} A_{c}^{2} A_{r}^{2} \cos \left(4 \pi f_{r} t\right) \\
&+\frac{1}{4} A_{c}^{2} A_{r}^{2}\left[\cos \left(\left(4 \pi f_{r}-4 \pi f_{c}\right) t\right)+\cos \left(\left(4 \pi f_{r}+4 \pi f_{c}\right) t\right)\right] \\
&+\operatorname{Bias} \times A_{c} A_{r}^{2}\left[\cos \left(\left(4 \pi f_{r}+2 \pi f_{c}\right) t\right)+\cos \left(\left(4 \pi f_{r}-2 \pi f_{c}\right) t\right)\right] \\
&+2 \operatorname{Bias}^{2} A_{c} A_{r}^{2} \cos \left(2 \pi f_{c} t\right)+\operatorname{Bias}^{2} A_{r}^{2} \cos \left(4 \pi f_{r} t\right) \\
&+\operatorname{Bias}^{2} A_{r}^{2} .
\end{aligned}
$$

A low-pass filter with a cut-off frequency lower than $4 \pi$ $\left(f_{r}-f_{c}\right)$ is used to filter out the high-frequency components of $V^{\prime}(t)$. Afterward, the signal components with frequencies of $4 \pi f_{r}, 4 \pi f_{r}-2 \pi f_{c}, 4 \pi f_{r}+2 \pi f_{c}$, and $4 \pi\left(f_{r}+f_{c}\right)$ are all filtered out. The signal with the retained frequency components is expressed as follows:

$$
\begin{aligned}
V^{\prime \prime}(t)= & A_{c}^{2} A_{r}^{2} \cos ^{2}\left(2 \pi f_{c} t\right)+2 \operatorname{Bias} \times A_{c} A_{r}^{2} \cos \left(2 \pi f_{c} t\right) \\
& +\operatorname{Bias}^{2} A_{r}^{2} \\
= & A_{r}^{2}\left(A_{c} \cos \left(2 \pi f_{c} t\right)+\text { Bias }\right)^{2} .
\end{aligned}
$$

Square root operation is applied to $V^{\prime \prime}(t)$, and then the demodulated signal is expressed as follows:

$$
\begin{aligned}
V_{d}(t) & =\operatorname{sqrt}\left(V^{\prime \prime}(t)\right) \\
& =A_{r}\left(A_{c} \cos \left(2 \pi f_{c} t\right)+\text { Bias }\right)
\end{aligned}
$$

Only the low-frequency $f_{c}$ is retained after signal demodulation, as shown in (6). Hence, the demodulated signal can be downsampled to reduce the signal length. 


\section{Signal Downsampling and Mixing}

The processed vibration and trigger signals are downsampled to reduce the IIoT transmitted data. Considering that the trigger signal is sparse and has definite amplitude, the demodulated vibration signal $V_{d}[n]$ and trigger signal $\operatorname{Tr} i[n]$ are mixed and then transmitted according to Algorithm 2.

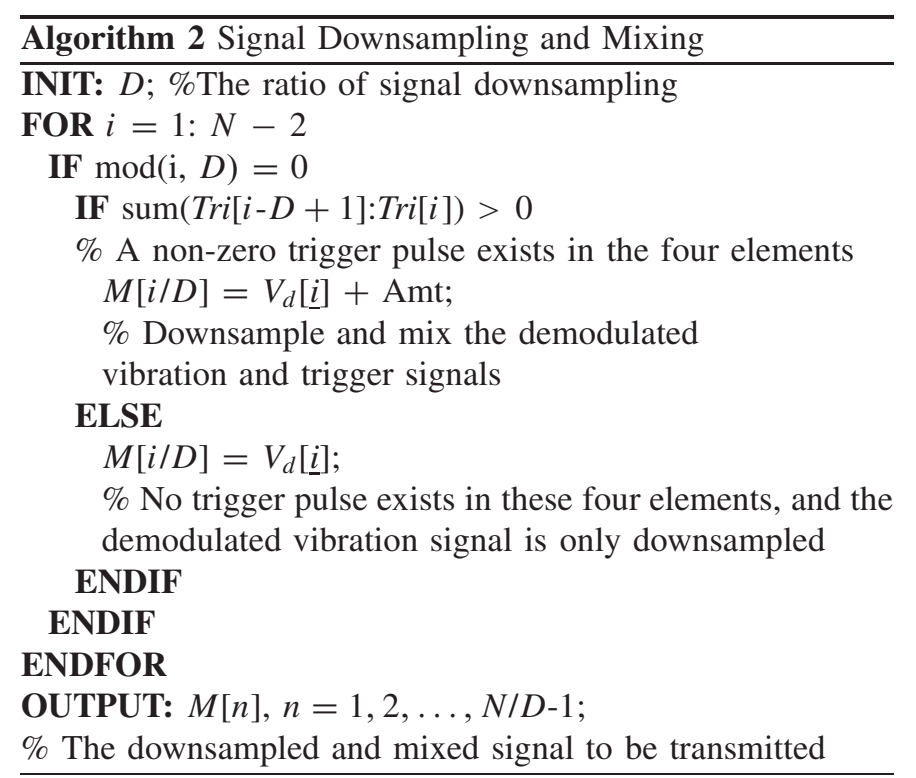

In the signal mixing procedure, the amplitude of the trigger signal should be higher than that of the maximal amplitude of the demodulated vibration signal as follows:

$$
\text { Amt }>\max \left(V_{d}[n]\right) .
$$

By applying Algorithm 2, the data length is reduced with a ratio of $D$, and the IIoT transmission time and power reduce accordingly.

\section{Signal Separation and Fault Diagnosis on Server}

The mixed signal is then transmitted from the IIoT node to the server to realize bearing fault diagnosis. The received mixed signal is denoted as $M[n], n=1,2, \ldots, N / D-1$. The mixed signal is separated by a threshold value $T h$ that satisfies the following:

$$
\text { Amt }>T h>\max \left(V_{d}[n]\right) .
$$

Afterward, the separated demodulated vibration signal $V_{d s}[n]$ and the trigger signal Tris $[n]$ are recovered according to Algorithm 3.

The rotation angle curve is calculated from the trigger signal that records the key phases of the leakage flux signal of the PMSM. The resolution of the mechanical angle, that is, AR, has been calculated in (2). When the PMSM spins AR degrees, a nonzero pulse can be detected in the trigger signal. Hence, the rotation angle curve is constructed from the trigger signal along the time axis using Algorithm 4.

In practice, the length of the cumulative signal $J$ is shorter than that of the processed vibration signal $N / D-1$. The length of the cumulative angle signal should be extended to
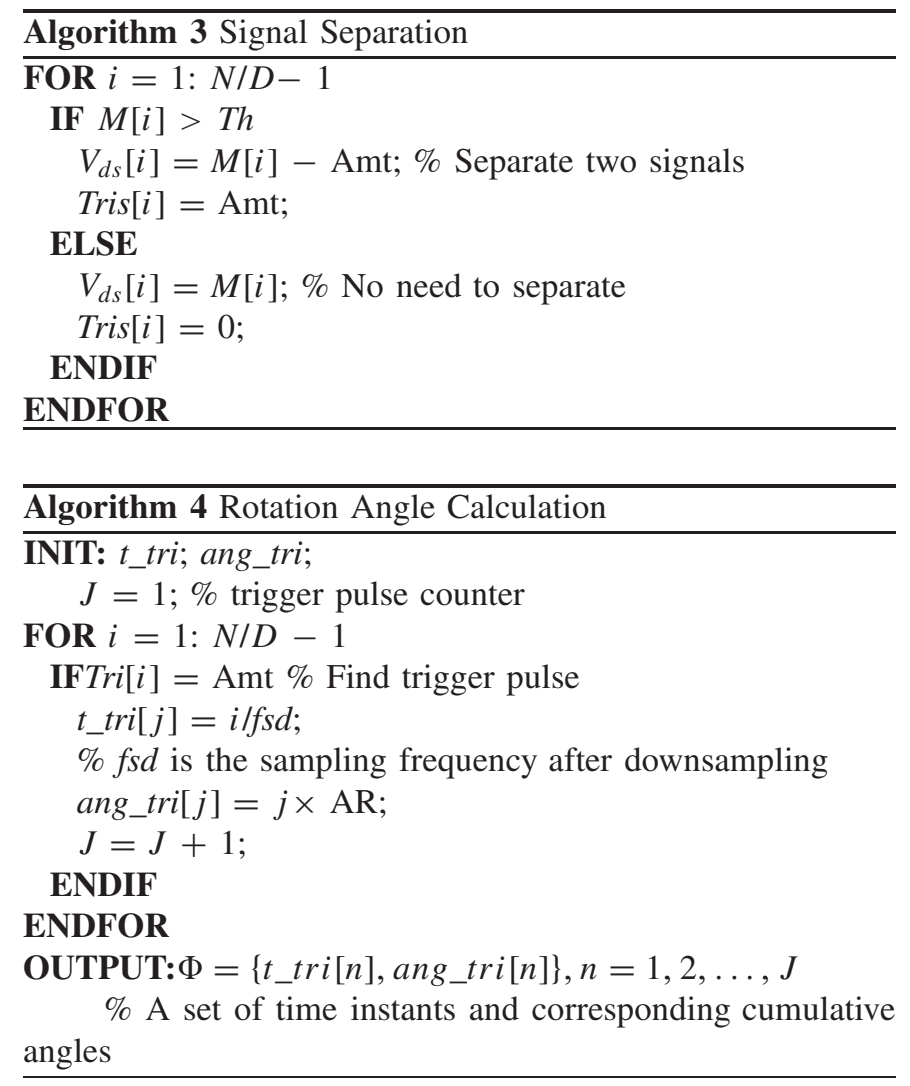

$N / D-1$ to resample the time-domain vibration signal and obtain the angular-domain signal. The time instants of the downsampled vibration signal are denoted as follows:

$$
\Psi=\left\{t_{d}[n]\right\}, \quad n=1,2, \ldots, N / D-1 .
$$

The extended cumulative angle signal is obtained as follows:

$$
\begin{aligned}
\mathrm{A} & =\{\operatorname{Ang}[n], n=1,2, \ldots, N / D-1\} \\
& =\text { interp1 }\left(t \text { tri }[\cdot], \text { ang_tri }[\cdot], t_{d}[\cdot],{ }^{\prime} \text { spline }^{\prime}\right)
\end{aligned}
$$

where interp1() is the numerical interpolation function in MATLAB, and spline represents the interpolation method. Afterward, the vibration signal is resampled using the extended angle signal as follows [25]:

$$
\begin{aligned}
& V_{d r}[n] \\
& =\operatorname{resample}(V d[n], \operatorname{Ang}[n]), \quad n=1,2, \ldots, N / D-1
\end{aligned}
$$

where resample() is the signal resampling function in MATLAB. The spectrum of the signal $V_{d r}[n]$ is calculated using fast Fourier transform (FFT), and then the bearing fault is revealed by the signatures in the spectrum.

\section{EXPERIMENTAL SETUP}

An experimental setup is designed to validate the performance of the proposed data reduction method for IIoT-based PMSM bearing fault diagnosis, as shown in Fig. 6(a). The test PMSM drives a permanent magnet synchronous generator (PMSG) as the mechanical load. The three-phase outputs of the PMSG connect to a resistor network as the electrical load. The PMSM is driven by a servo controller and rotates 


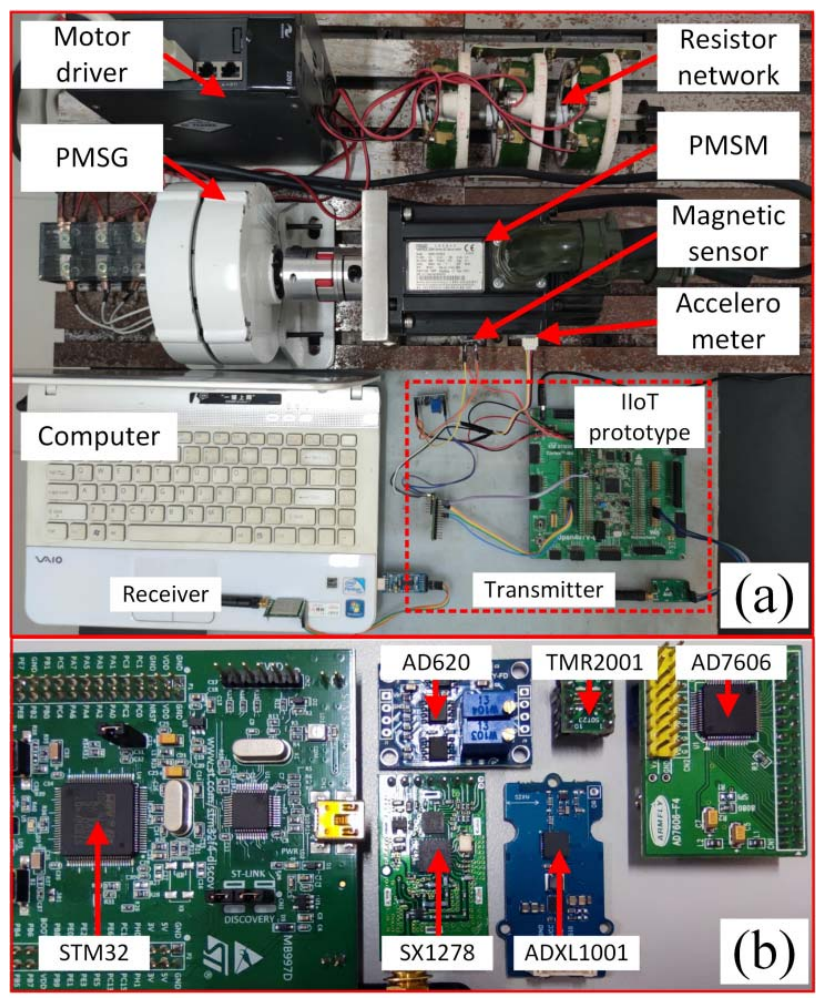

Fig. 6. (a) Experimental setup. (b) MCU and sensor modules.

TABLE I

PMSM PARAMETERS

\begin{tabular}{cccccc}
\hline \hline Type & $\begin{array}{c}\text { Rated } \\
\text { voltage }\end{array}$ & $\begin{array}{c}\text { Rated } \\
\text { torque }\end{array}$ & $\begin{array}{c}\text { Rated } \\
\text { power }\end{array}$ & $\begin{array}{c}\text { No. of } \\
\text { pole pairs }\end{array}$ & $\begin{array}{c}\text { No. of } \\
\text { phases }\end{array}$ \\
\hline ISMH2 & $220 \mathrm{VAC}$ & $3.18 \mathrm{Nm}$ & $1 \mathrm{~kW}$ & 4 & 3 \\
\hline \hline
\end{tabular}

at variable speed conditions. The bearing on the encoder end is used for tests.

The accelerometer is installed on the motor housing at the radial plane of the bearing using epoxy glue. The TMR linear sensor is installed on the center area of one side of the motor housing using epoxy glue. The two signals are acquired and processed by the IIoT prototype and then transmitted to a computer. The theoretical transmission distance between the transmitter and receiver is $1000 \mathrm{~m}$. To display all the components in one image, the transmitter and receiver are placed closely in Fig. 6(a). The sampling frequency and duration are set as $10 \mathrm{kHz}$ and $2 \mathrm{~s}$, respectively. The length of the signal, that is, $N$, is obtained as 20000 . For fast prototyping, the IIoT node is built from multiple MCU and sensor modules as shown in Fig. 6(b).

The parameters of the PMSM and test bearing are shown in Tables I and II, respectively. An artificial fault with a line type and a size of $0.3 \mathrm{~mm}$ (width) $\times 1 \mathrm{~mm}$ (depth) is set on the outer raceway fault of the test bearing. A spot type fault with a radius of $0.5 \mathrm{~mm}$ is set on the inner raceway of another test bearing. The line type fault and spot type fault can be regarded as a severe and a slight fault, respectively. When the rollers roll
TABLE II

BEARING PARAMETERS

\begin{tabular}{ccccccc}
\hline \hline Type & $\begin{array}{c}\text { Outside } \\
\text { diameter }\end{array}$ & $\begin{array}{c}\text { Inside } \\
\text { diameter }\end{array}$ & $\begin{array}{c}\text { No. of } \\
\text { rollers }\end{array}$ & $\begin{array}{c}\text { Contact } \\
\text { angle }\end{array}$ & FCO & FCO II \\
\hline 6203 & $40 \mathrm{~mm}$ & $17 \mathrm{~mm}$ & 8 & $0^{\circ}$ & 3.055 & 4.945 \\
\hline \hline
\end{tabular}
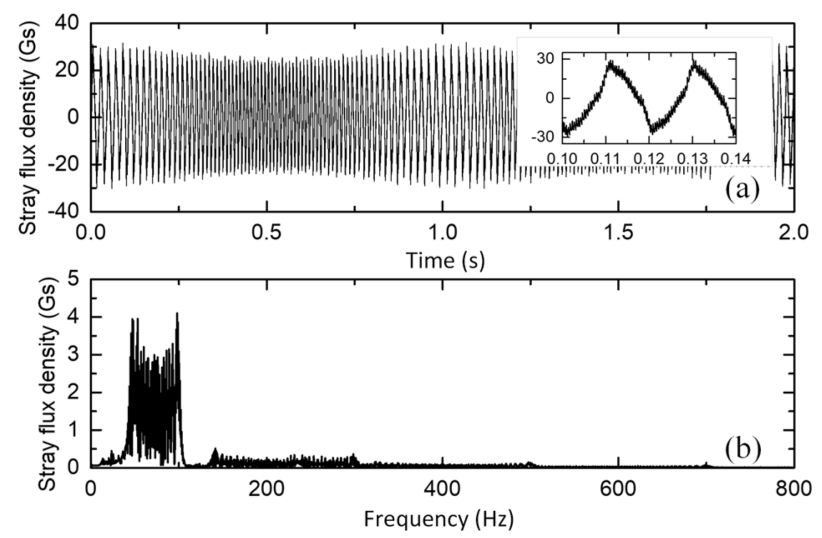

Fig. 7. Acquired magnetic signals. (a) Waveform and (b) amplitude spectrum.

over the defect point, the impulses among the mechanical parts appear. The number of impulses in one revolution is known as fault characteristic order (FCO). The FCO at the outer and inner raceway faults is calculated according to the following equations as [32]

$$
\begin{gathered}
\mathrm{FCO}_{\mathrm{O}}=\frac{N_{r}}{2}\left(1-\frac{d_{r}}{d_{p}} \cos \gamma\right) \\
\mathrm{FCO}_{\mathrm{I}}=\frac{N_{r}}{2}\left(1+\frac{d_{r}}{d_{p}} \cos \gamma\right)
\end{gathered}
$$

where $N_{r}$ is the number of the rollers, $d_{r}$ and $d_{p}$ are the roller and pitch diameters, respectively, and $\gamma$ is the contact angle. The $\mathrm{FCO}_{\mathrm{O}}$ and $\mathrm{FCO}_{\mathrm{I}}$ are calculated as 3.055 and 4.945, respectively, as shown in Table II.

\section{EXPERIMENTAL RESUlts}

The effectiveness of the proposed data reduction method and the performance of the designed IIoT node are evaluated in this section.

\section{A. Results of Magnetic Signal Processing}

In the experiments, the PMSM speed is set to fluctuate within a range of 600-1500 rpm. The motor accelerates and decelerates with a period of $1 \mathrm{~s}$. The magnetic signal acquired by the TMR sensor is shown in Fig. 7(a). The amplitude and frequency of the signal change with the variation in the rotation speed. As the leaked flux propagates through the stator laminations and motor housing, the signal is corrupted by background noises, as shown in the zoomed-in figure in Fig. 7(a). The spectrum of the magnetic signal indicates that the frequency distributes at around $40-100 \mathrm{~Hz}$. The high-frequency interference at $130-500 \mathrm{~Hz}$ is also noticeable in the spectrum. 


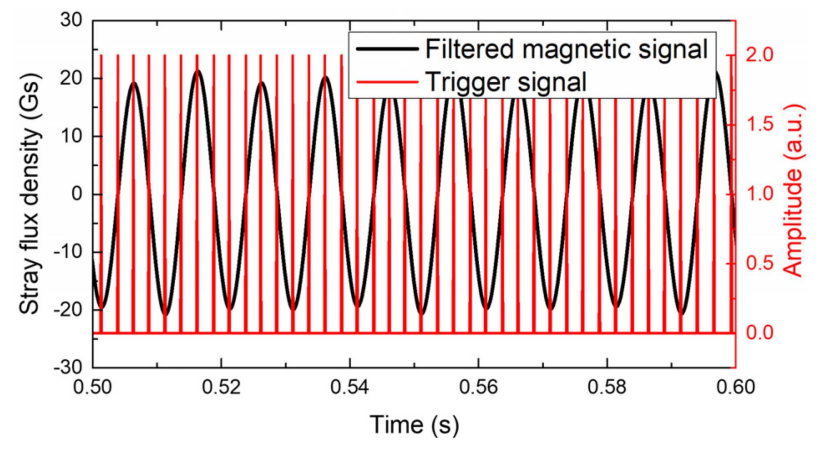

Fig. 8. Filtered magnetic and generated trigger signals.
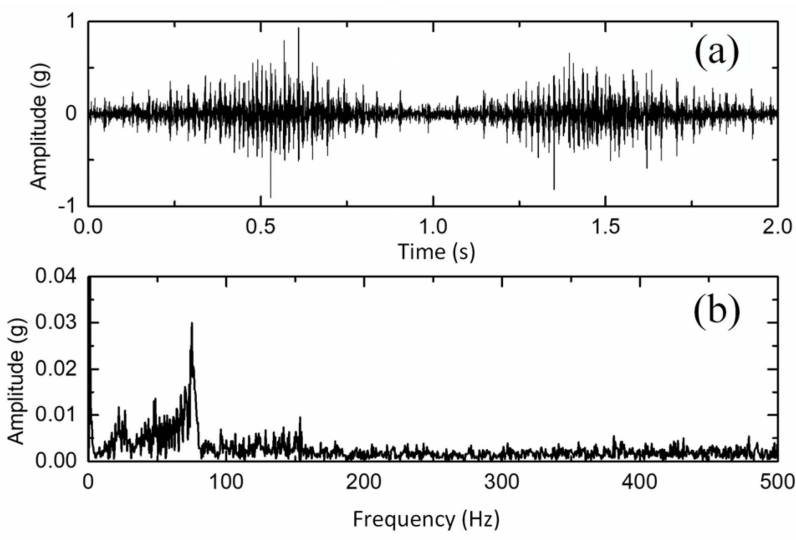

Fig. 9. Vibration signals. (a) Waveform and (b) envelope spectrum.

A third-order Butterworth bandpass filter with a bandwidth of $20-120 \mathrm{~Hz}$ is applied to the magnetic signal to reduce the noise interference. The high-frequency noise has been eliminated, as shown by the partially filtered signal in Fig. 8. Subsequently, the key phases of the filtered magnetic signal are positioned according to Algorithm 1, and the results are presented in Fig. 8. The amplitude of the trigger signal, that is, Amt, is set as 2. The key phases in each signal, that is, $\pi / 2, \pi, 3 \pi / 2$, and $2 \pi$, have been correctly positioned.

\section{B. Results of Vibration Signal Processing}

The synchronous sampled vibration signal acquired by the MEMS accelerometer is shown in Fig. 9(a). Similar to the magnetic signal, the amplitude and frequency of the vibration signal are changed with the motor speed. Amplitude demodulation is applied to the vibration signal to reveal the fault features in the low-frequency region. The envelope spectrum of the demodulated signal is shown in Fig. 9(b). As the FFT is only suitable for processing the stationary signal, the frequency smearing phenomenon appears in the envelope spectrum. Specifically, no explicit frequency bins can reveal the bearing fault type.

\section{Results of Signal Mixing and Separation}

The processed rotation speed and vibration signals are transmitted from the IIoT to the server to diagnose the PMSM bearing fault under variable speed conditions. Then, the trigger
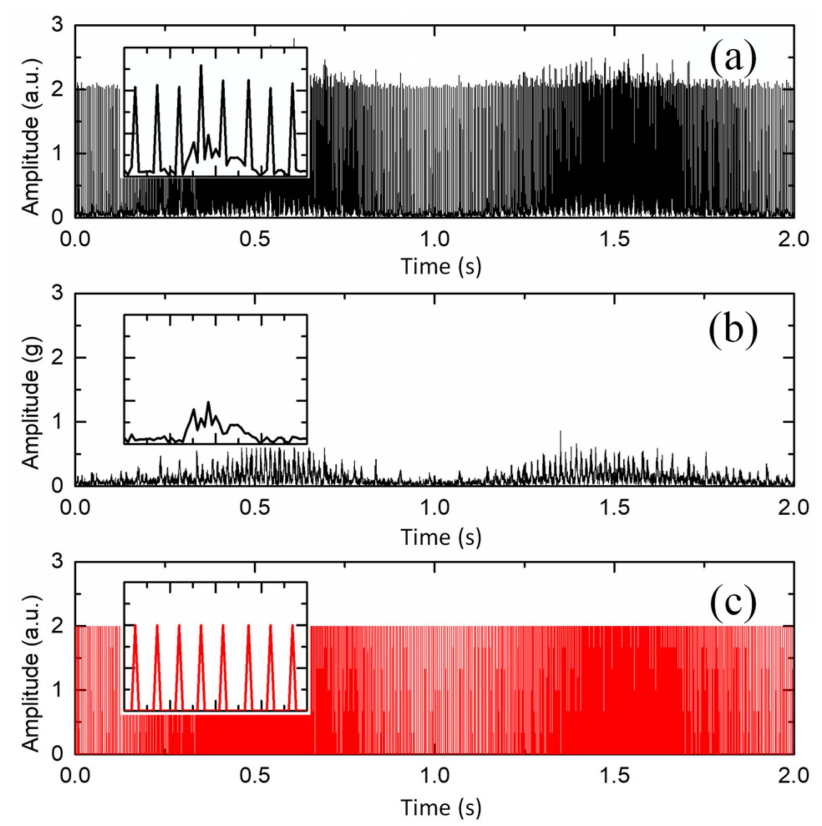

Fig. 10. (a) Mixed vibration and trigger signals, (b) recovered vibration signal, and (c) recovered trigger signal.

and demodulated signals are downsampled with a ratio $D=4$ to reduce the length of the transmitted signal. As the trigger signal is a sparse signal and its amplitude is a constant value, it is mixed with the demodulated signal before signal transmission. The vibration signal amplitude is smaller than 1 , as shown in Fig. 9(a). According to (7), the amplitude of the trigger signal is set as 2 to guarantee the signal separation. The mixed demodulated vibration and trigger signals are shown in Fig. 10(a). The amplitude range of the mixed signal is $0-3$, and the profiles of the trigger signal with fixed amplitude can be noticed. The mixed signal from the IIoT node is received by a server and then separated according to Algorithm 3. According to (8), the threshold $T h$ for signal separation is set as 1.5. The separated demodulated vibration signal and trigger signal are shown in Fig. 10(b) and (c), respectively. The figures and the zoomed-in figures indicate that these two signals have been correctly separated and recovered. Thus, the effectiveness of the proposed data reduction method is validated.

\section{Results of PMSM Bearing Fault Diagnosis}

The cumulative rotation angle of the motor shaft is calculated from the trigger signal according to Algorithm 4, and the result is shown in Fig. 11(a). The number of pole pairs of the PMSM is 4, and four key phases in each period are extracted. Hence, the angular resolution is $\mathrm{AR}=22.5^{\circ}$. The cumulative angle increases with time in a nonlinear mode due to speed variation. The demodulated signal is then resampled using the angle curve, and the result is shown in Fig. 11(b). The intervals of the fault-induced impulses tend to be consistent after signal resampling. The spectrum of the resampled signal is calculated and shown in Fig. 11(c). The fault signatures $\mathrm{FCO}_{\mathrm{O}}$ and its second harmonic are observed in the spectrum, 

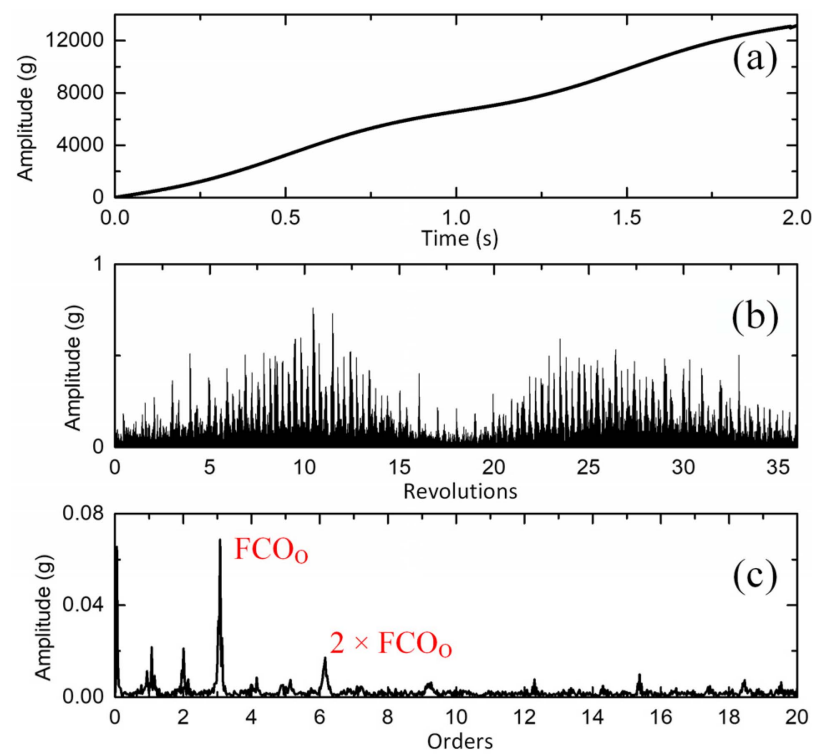

Fig. 11. (a) Calculated rotation angle signal, (b) resampled vibration signal, and (c) envelope order spectrum.

thereby confirming that the tested bearing is subjected to an outer raceway fault. The above results demonstrate that the designed IIoT node and the data reduction algorithm embedded can diagnose PMSM bearing fault under variable speed conditions.

\section{Vi. Performance Evaluation of THE PRoposed Method}

To demonstrate the advantages of the proposed method, a series of comparative experiments in considering of both algorithm and hardware are conducted.

\section{A. Performance Comparison With a Traditional Method Using Two IIoT Nodes}

A traditional method is used for comparison. In the traditional method, two IIoT nodes are used for encoder signal and vibration signal acquisitions, respectively. Given that the motor driver and the motor housing have a certain distance, two nodes are respectively installed on them. The resolution of the optical encoder is 2500 pulses per revolution, and the maximal rotation speed is approximately $25 \mathrm{~Hz}$ (number of pole pairs $=4$ ) in the experiment, as shown in Fig. 7(b). Hence, the maximal frequency of the encoder is approximately $62.5 \mathrm{kHz}$. Accordingly, the sampling frequency of the IIoT node for encoder signal acquisition is set as $200 \mathrm{kHz}$. A rising edge detection algorithm is applied to localize the pulses, and the pulses' positions are transmitted to the receiver for rotation angle calculation. The sampling frequency of the vibration signal is set similar to that of the proposed method, that is, $10 \mathrm{kHz}$. The two IIoT nodes are synchronously triggered by a command from the server.

The sampling duration is set as $2 \mathrm{~s}$. In the test of the bearing with outer raceway fault, a total of 90540 pulses are detected in the encoder signal with a length of 400000 points. The lowest five decimal digits of each pulse's location are transmitted
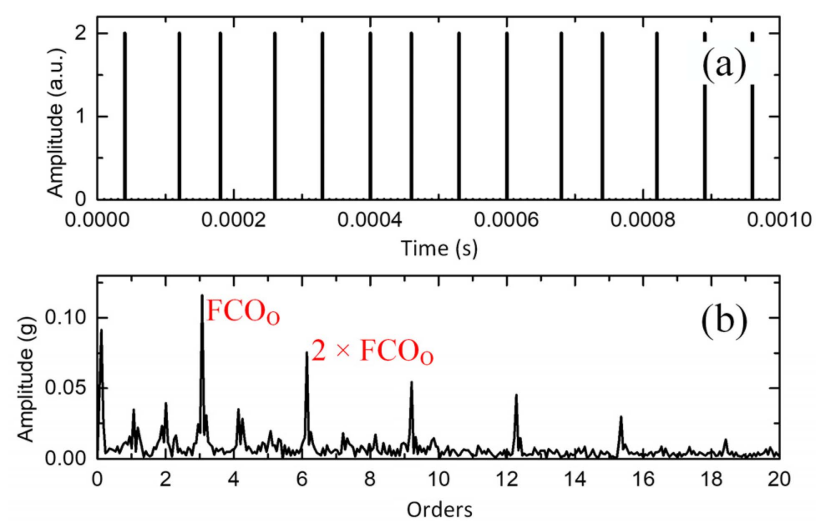

Fig. 12. (a) Acquired encoder signal using another IIoT node and (b) envelope order spectrum using the encoder signal.

in a 2 bytes hexadecimal format. The highest decimal digit increases automatically in every million cycles. Afterward, a total of 181080 bytes encoder data and 40000 bytes vibration data are transmitted. Partially received pulse signals are shown in Fig. 12(a). The vibration signal is demodulated and then resampled using the cumulative angle curve calculated from the encoder signal, and the result is shown in Fig. 12(b). The fault signatures $\mathrm{FCO}_{\mathrm{O}}$ and its multiple harmonics are revealed in the spectrum. These results are similar to those in Fig. 11(c) generated by the proposed method. Here, the signal-to-noise ratio (SNR) index is used to evaluate the error of signal resampling and the accuracy of fault diagnosis as follows [25]:

$$
\begin{aligned}
& \mathrm{SNR}=10 \log _{10} \\
& \times \frac{\left.\left.\sum_{k=1}^{5} A E k \times \text { round } \frac{\mathrm{FCO} \times N}{f_{s}}\right)+1\right]}{\left.\sum_{h=0}^{N / 2-1} A E[h]-\sum_{k=1}^{5} A E k \times \text { round } \frac{\mathrm{FCO} \times N}{f_{s}}+1\right]}
\end{aligned}
$$

where $A E[h], h=0,1, \ldots, N / 2-1$ is the magnitude of the frequency bin in the envelope order spectrum. The FCO and its second to fifth harmonics are supposed to be the useful signals and the other components are noises. The SNR values of the proposed and traditional method are, respectively, computed as -5.61 and $-4.89 \mathrm{~dB}$, as shown in the rightmost column in Table III. These two values are close to each other, thereby indicating that both the proposed and traditional methods can effectively diagnose the PMSM bearing fault under variable speed conditions.

The transmission data volume, transmission time, and computation time of two methods are evaluated and summarized in Table III. In the experiment of bearing outer raceway fault, the total data length of the traditional method is 221080 bytes, including the encoder and vibration signals. In contrast, the total data length is only 10000 bytes for the proposed method, as each data point occupies 2 bytes storage space. The transmission time is measured and shown in the 7th column of Table III. It can be seen that the transmission time is approximately in direct proportion to the data volume, because the transmission rate is fixed. The comparison results indicated that the proposed method reduces about $95 \%$ of the transmission data and time while achieving 
TABLE III

Comparison of the Proposed And Traditional Methods ${ }^{\#}$

\begin{tabular}{|c|c|c|c|c|c|c|c|c|c|c|}
\hline Method & $\begin{array}{c}\text { Fault } \\
\text { location }\end{array}$ & $\begin{array}{l}\text { Fault } \\
\text { shape }\end{array}$ & $\begin{array}{l}\text { Fault size } \\
(\mathrm{mm})\end{array}$ & $\begin{array}{l}\text { Max freq. } \\
(\mathrm{kHz})\end{array}$ & $\begin{array}{c}\text { Sampling } \\
\text { time (s) }\end{array}$ & $\begin{array}{c}\text { Sampling } \\
\text { freq. }(\mathrm{kHz})^{*}\end{array}$ & $\begin{array}{l}\text { Processing } \\
\text { time }(\mathrm{s})\end{array}$ & $\begin{array}{l}\text { Transmission } \\
\text { time (s) }\end{array}$ & $\begin{array}{c}\text { Transmission } \\
\text { data }(\mathrm{KB})\end{array}$ & SNR (dB) \\
\hline Prop. & Outer & Line & $0.3 \times 1$ & 200 & 2.00 & 10 & 1.79 & 11.13 & 10.00 & -5.61 \\
\hline Trad. & Outer & Line & $0.3 \times 1$ & 200 & 2.00 & $10 / 200$ & - & 245.87 & 221.08 & -4.89 \\
\hline Prop. (10 tests) & Inner & Spot & $\mathrm{r}=0.5$ & 200 & 2.00 & 10 & $1.82 \pm 0.21$ & $11.43 \pm 0.45$ & 10.00 & $-7.44 \pm 0.41$ \\
\hline Trad. (10 tests) & Inner & Spot & $\mathrm{r}=0.5$ & 200 & 2.00 & $10 / 200$ & - & $225.62 \pm 3.76$ & $204.64 \pm 10.62$ & $-7.03 \pm 0.27$ \\
\hline
\end{tabular}

${ }^{\text {" }}$ Prop. = Proposed, Trad. = Traditional, freq. = frequency; ${ }^{*} 10 \mathrm{kHz}$ for vibration signal and $200 \mathrm{kHz}$ for encoder signal in the traditional method.

comparable bearing fault diagnosis performance. In addition, the computation time of the proposed method is measured as shown in the 6th column of Table III. The total time for signal processed, downsampled, and mixed is $1.79 \mathrm{~s}$ on the IIoT node. Such a result is acceptable considering that the sampling time is $2 \mathrm{~s}$.

To further evaluate the performances of the proposed method, the bearing with a small-size inner raceway fault is tested. Considering that the uncertainties and errors are induced from the time-varying signals, mechanical noises, and electrical noises from the circuits, the experimental results are averaged from ten times of tests and the error ranges are calculated. The results are summarized in the 4th and 5th rows in Table III. It can be seen that processing time, transmission time, and data of the inner raceway fault experiments are close to those of the outer ones for the proposed or traditional method. Because the motor rotation speed varies in each test, the detected key rotation phases' information varies accordingly, and finally the processing and transmission time vary in the specific ranges.

Besides, it can be seen from the rightmost column that the SNR values of the inner raceway fault experiments are lower than those of the outer ones. Such results are caused by a fact that the fault size on the inner raceway is smaller than that on the outer raceway. Hence, the inner raceway fault signal is weak. Even so, all the envelope order spectra have been carefully checked, and the fault indicators $\mathrm{FCO}_{\mathrm{I}}$ and its harmonics can be clearly identified in the spectra. In other words, the proposed method is still reliable for bearing fault diagnosis with small fault size.

\section{B. Current Consumptions in Busy and Idle Stages}

To further evaluate the performance of the IIoT node, the current consumption curves at busy and idle stages are measured and plotted in Fig. 13(a). The IIoT node is powered by a voltage regulator with the output set as $5 \mathrm{~V}$. The busy stage can be divided into three regions including signal acquisition, signal processing, and signal transmission, as indicated by different background colors in Fig. 13(a). Because different circuit components work at different stages, the consumed currents vary accordingly. The zoomed-in figure of the signal transmission stage is plotted within Fig. 13(a). The transmitter module collects the data, constructs the data packets, and then transmits them frame by frame, and hence it can be seen from the zoomed-in curve that the current consumption is fluctuated. After signal transmission, the MCU is configured into the
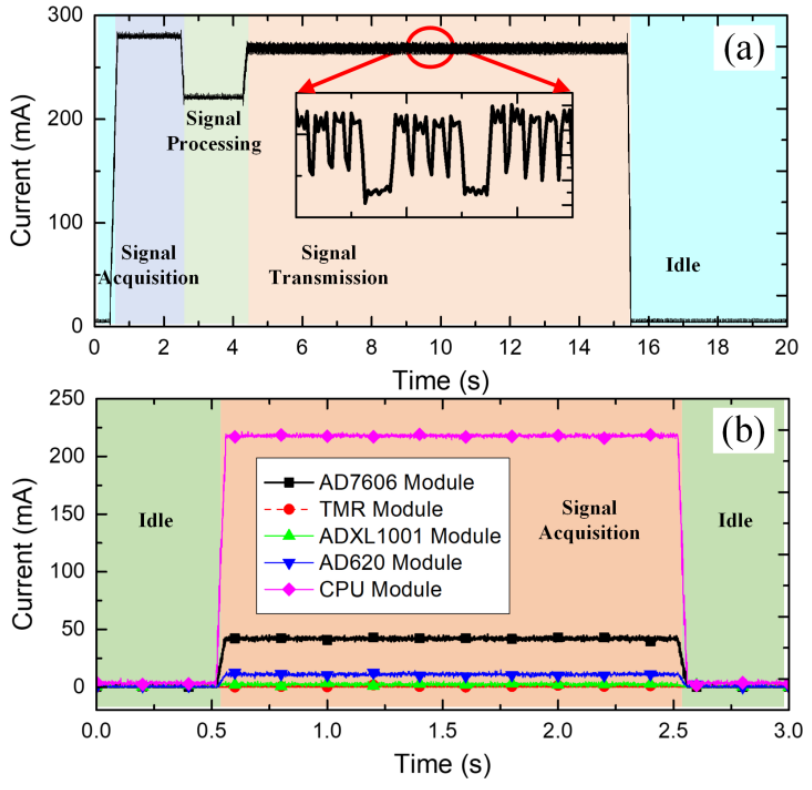

Fig. 13. (a) Current consumption of the proposed method in different stages and (b) current consumption of the CPU and sensor modules in different stages.

standby stage and the sensors are turned off by electrical switches to reduce current consumption.

In addition, the current consumption curves of the CPU and sensor modules in the acquisition and idle stages are also measured and plotted in Fig. 13(b). It can be seen that the CPU module consumes much more current than the sensors in the signal acquisition stage. How to reduce the current consumption of the IIoT node will be further discussed in Section VII.

\section{Performance Evaluation of the IIoT Nodes With Different Hardware Configurations}

The IIoT nodes that can perform edge computing require sufficient hardware resources and battery capacities. In this subsection, the proposed data reduction algorithm and the traditional algorithm are separately transplanted to another STM32WB platform with lower CPU frequency. Additionally, different wireless communication protocols are also tested in the experiments to estimate the battery life. The battery life is calculated based on a commonly used IIoT Li-battery with type ER34615 (EVE Energy Inc. China, price: 10 USD). The parameters of the battery are shown as follows: 
TABLE IV

Evaluation of the Hardware Platform With Different Configurations

\begin{tabular}{cccccccc}
\hline \hline Method & CPU Type & $\begin{array}{c}\text { CPU freq. } \\
(\mathrm{MHz})\end{array}$ & $\begin{array}{c}\text { Wireless } \\
\text { protocol }\end{array}$ & $\begin{array}{c}\text { Max transmission } \\
\text { distance }(\mathrm{m})\end{array}$ & $\begin{array}{c}\text { Consumed } \\
\text { time (s) }\end{array}$ & $\begin{array}{c}\text { Consumed } \\
\text { energy (J) }\end{array}$ & $\begin{array}{c}\text { Est. battery } \\
\text { life (month) }\end{array}$ \\
\hline Proposed (10 tests) & STM32F4 & 168 & LoRa & 1000 & $14.83 \pm 0.52$ & $19.17 \pm 0.94$ & 3.81 \\
Traditional (10 tests) & STM32F4 & 168 & LoRa & 1000 & $242.54 \pm 5.21$ & $328.42 \pm 6.18$ & 0.86 \\
Proposed (10 tests) & STM32WB & 64 & Bluetooth & 10 & $62.43 \pm 1.45$ & $6.78 \pm 0.63$ & 11.79 \\
Traditional (10 tests) & STM32WB & 64 & Bluetooth & 10 & $1071.71 \pm 7.65$ & $116.39 \pm 4.35$ & 2.57 \\
\hline \hline
\end{tabular}

diameter $=34.2 \mathrm{~mm}$, height $=61.5 \mathrm{~mm}$, capacity $=19 \mathrm{AH}$, voltage $=3.6 \mathrm{~V}$, and total energy $=246240 \mathrm{~J}$ [33]. The signal is assumed to be acquired and transmitted in a period of 1 hour, namely, 24 signal segments are transmitted in one day. For simplicity, the state of charge (SoC) of the battery is not taken into account in this study. The estimated battery life (EBL) is calculated using the equation as

$$
\operatorname{EBL}(\text { month })=\frac{\text { Battery Total Energy }}{\sum_{i=1}^{4} V_{i} I_{i} T_{i} \times 24 \frac{\text { hours }}{\text { day }} \times 30 \frac{\text { day }}{\text { month }}}
$$

in which $V_{i}, I_{i}$, and $T_{i}$ are the averaged voltage, averaged current, and duration of different stages, respectively. $i=1$, 2, 3, 4 represents the signal acquisition, signal processing, signal transmission, and idle stages, respectively.

The comparative results are summarized in Table IV. It can be seen that the proposed data reduction algorithm can be executed on the hardware platform with 64-MHz CPU frequency. For a fixed transmission data volume, the total consumed time, energy, and estimated battery life are largely dominated by the wireless transmission power and protocol. Even so, the proposed data reduction method obviously extends the battery life in the same hardware platform when compared with the traditional method as shown in the rightmost column in Table IV.

\section{DISCUSSION}

Edge computing is a promising technique for real-time motor condition monitoring, fault diagnosis, and dynamic control. To improve the flexibility and efficiency of the proposed method in industrial applications, some factors affecting the method performance are discussed.

\section{A. Improvement of Fault Diagnosis Accuracy}

This study focuses on the preprocessing step of motor bearing fault diagnosis, namely, signal acquisition and data reduction. In practice, the acquired signals are easily subjected to mechanical and electrical noise interferences. In this regard, the techniques such as wavelet analysis [34] and stochastic resonance [35] can be used to filter or enhance the weak signal to improve the output SNR. With the rapid increase in the computation power of an IIoT node, the signal filtering and enhancing algorithms can be optimized and implemented on the nodes to improve the accuracy and robustness of motor fault diagnosis in real time.

\section{B. Improvement of Hardware Performance and Power Efficiency}

In recent years, hardware advance of an IIoT node makes the execution of a complicated algorithm become practical. Considering that some IIoT nodes are battery-powered, improving the power efficiency is of significance. As demonstrated in the results in Table IV, signal transmission is rather energy-consumed in all the procedures, especially for long-distance signal transmission. Thus, how to optimize wireless transmission including protocol, codec, data packet, and error rate deserves a further investigation. Combining the proposed data reduction algorithm and the optimized wireless communication will improve the motor fault diagnosis efficiency and extend the battery life simultaneously, which will be further studied in the future.

\section{Using the Proposed Method for Other Motors}

In this study, the rotation speed of the PMSM is estimated from the leakage flux signal. The speed of the rotor strictly synchronizes with that of the stator rotational magnetic field. The proposed method is also suitable for other synchronous motors such as a brushless direct current motor. For an induction motor, if the slip rate between the stator and rotor is known, the leakage flux signal analysis method can also be used for rotation speed measurement. In sum, the proposed method shows potential applications for fault diagnosis of other types of motors after proper modifications.

\section{Commercialization of IIoT Nodes}

In this study, the IIoT node prototype is assembled from different commercial circuit modules for convenience. It should be noted that these modules have many redundant components including variable resistors, LEDs, and additional CPUs for program download and chip control. If the IIoT node is commercialized for practical application, a printed circuit board (PCB) integrating all the necessary components should be re-designed. A power management chip, a battery management system considering the battery SoC, and an operation/sleep/stop/standby strategy should also be carefully designed to improve the PCB's energy efficiency and to extend the battery life. In addition, energy harvesting has become a hot research topic in recent years [36]. This technique can also be introduced into the IIoT node to realize self-power in the future, which will further extend the service life of the IIoT node. 


\section{CONCLUSION}

An efficient data reduction method is proposed and implemented on a designed IIoT node for variable speed PMSM bearing fault diagnosis. A TMR sensor and an accelerometer are used to capture the motor leakage flux and vibration signals, respectively. These two signals are processed, downsampled, mixed, and transmitted from the IIoT node to a server. The received signal is separated and then the vibration signal is analyzed to recognize the bearing fault type. The performances of the proposed method and designed IIoT node have been validated by experiments in comparison to a traditional method. The comparative results indicate that the proposed method reduces about $95 \%$ of the transmission data while generating satisfactory performance in bearing fault diagnosis. As the proposed method can efficiently reduce the transmission data on an IIoT node, it is suitable to apply on remote condition monitoring and fault diagnosis of PMSM, especially when the IIoT node is battery-powered.

\section{REFERENCES}

[1] M.-C. Tsai and P.-J. Ko, "On-line condition monitoring of servo motor drive systems by HHT in industry 4.0," J. Chin. Inst. Eng., vol. 40, no. 7, pp. 572-584, Oct. 2017.

[2] G. Wang, M. Valla, and J. Solsona, "Position sensorless permanent magnet synchronous machine drives-A review," IEEE Trans. Ind. Electron., vol. 67, no. 7, pp. 5830-5842, Jul. 2020.

[3] A. Mohammed, J. I. Melecio, and S. Durovic, "Electrical machine permanent magnets health monitoring and diagnosis using an air-gap magnetic sensor," IEEE Sensors J., vol. 20, no. 10, pp. 5251-5259, May 2020.

[4] B. M. Ebrahimi, J. Faiz, M. Javan-Roshtkhari, and A. Z. Nejhad, "Static eccentricity fault diagnosis in permanent magnet synchronous motor using time stepping finite element method," IEEE Trans. Magn., vol. 44, no. 11, pp. 4297-4300, Nov. 2008.

[5] Y. Song, Y. Li, L. Jia, and M. Qiu, "Retraining strategy-based domain adaption network for intelligent fault diagnosis," IEEE Trans. Ind. Informat., vol. 16, no. 9, pp. 6163-6171, Sep. 2020.

[6] R. Yan, H. Sun, and Y. Qian, "Energy-aware sensor node design with its application in wireless sensor networks," IEEE Trans. Instrum. Meas., vol. 62, no. 5, pp. 1183-1191, May 2013.

[7] W. Shi, J. Cao, Q. Zhang, Y. Li, and L. Xu, "Edge computing: Vision and challenges," IEEE Internet Things J., vol. 3, no. 5, pp. 637-646, Oct. 2016.

[8] M. J. Jafarian and J. Nazarzadeh, "A TDF model in induction machines for loose bearing diagnosis by neutral voltage," IEEE Trans. Ind. Electron., vol. 67, no. 10, pp. 8155-8163, Oct. 2020.

[9] M. Yang, N. Chai, Z. Liu, B. Ren, and D. Xu, "Motor speed signature analysis for local bearing fault detection with noise cancellation based on improved drive algorithm," IEEE Trans. Ind. Electron., vol. 67, no. 5, pp. 4172-4182, May 2020.

[10] M. Xia, T. Li, T. Shu, J. Wan, C. W. de Silva, and Z. Wang, "A two-stage approach for the remaining useful life prediction of bearings using deep neural networks," IEEE Trans. Ind. Informat., vol. 15, no. 6, pp. 3703-3711, Jun. 2019.

[11] S. Nandi, H. A. Toliyat, and X. Li, "Condition monitoring and fault diagnosis of electrical Motors-A review," IEEE Trans. Energy Convers., vol. 20, no. 4, pp. 719-729, Dec. 2005.

[12] G.-Y. Lee et al., "Machine health management in smart factory: A review," J. Mech. Sci. Technol., vol. 32, no. 3, pp. 987-1009, 2018.

[13] P. Gangsar and R. Tiwari, "Signal based condition monitoring techniques for fault detection and diagnosis of induction motors: A stateof-the-art review," Mech. Syst. Signal Process., vol. 144, Oct. 2020, Art. no. 106908

[14] M. Sohaib and J.-M. Kim, "Fault diagnosis of rotary machine bearings under inconsistent working conditions," IEEE Trans. Instrum. Meas., vol. 69, no. 6, pp. 3334-3347, Jun. 2020.

[15] B. R. Nayana and P. Geethanjali, "Improved identification of various conditions of induction motor bearing faults," IEEE Trans. Instrum. Meas., vol. 69, no. 5, pp. 1908-1919, May 2020.
[16] M. K. Babouri, A. Djebala, N. Ouelaa, B. Oudjani, and R. Younes, "Rolling bearing faults severity classification using a combined approach based on multi-scales principal component analysis and fuzzy technique," Int. J. Adv. Manuf. Technol., vol. 107, nos. 9-10, pp. 4301-4316, Apr. 2020.

[17] D. Wang, Z. Peng, and L. Xi, "The sum of weighted normalized square envelope: A unified framework for kurtosis, negative entropy, gini index and smoothness index for machine health monitoring," Mech. Syst. Signal Process., vol. 140, Jun. 2020, Art. no. 106725.

[18] H. Zhang, E. Liu, B. Zhang, and Q. Miao, "RUL prediction and uncertainty management for multi-sensor system using an integrated data-level fusion and UPF approach," IEEE Trans. Ind. Informat., early access, Aug. 18, 2020, doi: 10.1109/TII.2020.3017194.

[19] R. Razavi-Far, M. Farajzadeh-Zanjani, and M. Saif, "An integrated classimbalanced learning scheme for diagnosing bearing defects in induction motors," IEEE Trans. Ind. Informat., vol. 13, no. 6, pp. 2758-2769, Dec. 2017.

[20] W. Li, S. Zhang, and S. Rakheja, "Feature denoising and NearestFarthest distance preserving projection for machine fault diagnosis," IEEE Trans. Ind. Informat., vol. 12, no. 1, pp. 393-404, Feb. 2016.

[21] D. Ganga and V. Ramachandran, "IoT-based vibration analytics of electrical machines," IEEE Internet Things J., vol. 5, no. 6, pp. 4538-4549, Dec. 2018.

[22] Z. Mo, H. Zhang, J. Wang, J. Wang, H. Fu, and Q. Miao, "Adaptive meyer wavelet filters for machinery fault diagnosis based on harmonic infinite-taxicab norm and grasshopper optimization algorithm," Proc. Inst. Mech. Eng. C, J. Mech. Eng. Sci., vol. 15, Nov. 2020, Art. no. 095440622097166, doi: 10.1177/0954406220971663.

[23] A. K. Junejo, W. Xu, C. Mu, M. M. Ismail, and Y. Liu, "Adaptive speed control of PMSM drive system based a new sliding-mode reaching law," IEEE Trans. Power Electron., vol. 35, no. 11, pp. 12110-12121, Nov. 2020.

[24] S. Chen, Y. Yang, X. Dong, G. Xing, Z. Peng, and W. Zhang, "Warped variational mode decomposition with application to vibration signals of varying-speed rotating machineries," IEEE Trans. Instrum. Meas., vol. 68, no. 8, pp. 2755-2767, Aug. 2019.

[25] S. Lu, R. Yan, Y. Liu, and Q. Wang, "Tacholess speed estimation in order tracking: A review with application to rotating machine fault diagnosis,' IEEE Trans. Instrum. Meas., vol. 68, no. 7, pp. 2315-2332, Jul. 2019.

[26] J. Wang, Y. Peng, and W. Qiao, "Current-aided order tracking of vibration signals for bearing fault diagnosis of direct-drive wind turbines," IEEE Trans. Ind. Electron., vol. 63, no. 10, pp. 6336-6346, Oct. 2016.

[27] The Information for Chip sx1278. Accessed: Dec. 27, 2020. [Online]. Available: www.semtech.com/products/wireless-rf/loratransceivers/sx 1278

[28] X. Liu, C. Liu, and P. W. T. Pong, "Velocity measurement technique for permanent magnet synchronous motors through external stray magnetic field sensing," IEEE Sensors J., vol. 18, no. 10, pp. 4013-4021, May 2018.

[29] S. K. Mitra, Digital Signal Processing: A Computer-Based Approach. New York, NY, USA: McGraw-Hill, 2011.

[30] S. Lu and X. Wang, "A new methodology to estimate the rotating phase of a BLDC motor with its application in variable-speed bearing fault diagnosis," IEEE Trans. Power Electron., vol. 33, no. 4, pp. 3399-3410, Apr. 2018.

[31] T. Wang and F. Chu, "Bearing fault diagnosis under time-varying rotational speed via the fault characteristic order (FCO) index based demodulation and the stepwise resampling in the fault phase angle (FPA) domain," ISA Trans., vol. 94, pp. 391-400, Nov. 2019.

[32] T. Wang, M. Liang, J. Li, and W. Cheng, "Rolling element bearing fault diagnosis via fault characteristic order (FCO) analysis," Mech. Syst. Signal Process., vol. 45, no. 1, pp. 139-153, Mar. 2014.

[33] The Information for Battery ER34615. Accessed: Dec. 27, 2020. [Online]. Available: www.evebattery.com/product/2.html?s=ER34615

[34] R. Yan, R. X. Gao, and X. Chen, "Wavelets for fault diagnosis of rotary machines: A review with applications," Signal Process., vol. 96, pp. 1-15, Mar. 2014.

[35] S. Lu, Q. He, and J. Wang, "A review of stochastic resonance in rotating machine fault detection," Mech. Syst. Signal Process., vol. 116, pp. 230-260, Feb. 2019.

[36] H. Liu, J. Zhong, C. Lee, S.-W. Lee, and L. Lin, "A comprehensive review on piezoelectric energy harvesting technology: Materials, mechanisms, and applications," Appl. Phys. Rev., vol. 5, no. 4, Dec. 2018, Art. no. 041306. 